\title{
The relation between habitual sleep duration and blood pressure values in Japanese male subjects
}

\author{
Hiroki Satoh · Jun Nishihira $\cdot$ Tatsuhiko Wada \\ Satoshi Fujii $\cdot$ Hiroyuki Tsutui
}

Received: 29 August 2012/ Accepted: 30 September 2012/Published online: 21 October 2012

(c) The Japanese Society for Hygiene 2012

\begin{abstract}
Background Previous studies have demonstrated that sleep duration is closely associated with metabolic risk factors. However, the relationship between habitual sleep duration and blood pressure values in Japanese population has not been fully established.

Methods We performed a cross-sectional study of 1,670 Japanese male subjects to clarify the relationship between habitual sleep duration and blood pressure values. The study subjects were divided into four groups $(<6,6-, 7-$, and $\geq 8 \mathrm{~h}$ ) according to their nightly habitual sleep duration.

Results The rate of subjects with $<6,6-, 7-$, and $\geq 8 \mathrm{~h}$ sleep duration was $12.0,37.6,38.2$, and $12.2 \%$, respectively. Compared with the group with 7-h sleep duration (referent), the $<6$ and $\geq 8 \mathrm{~h}$ groups had significantly greater systolic and diastolic blood pressure values. The rate of hypertensive subjects, defined as systolic blood pressure $\geq 140 \mathrm{mmHg}$ and/or diastolic blood pressure $\geq 90 \mathrm{mmHg}$, with sleep duration of $<6,6-, 7-$, and $\geq 8 \mathrm{~h}$ was $13.4,5.7$, 7.5 , and $13.8 \%$, respectively. Compared with the group with 7-h sleep duration (referent), the multivariate odds
\end{abstract}

H. Satoh $(\bowtie) \cdot$ J. Nishihira $~ T$. Wada

Department of Clinical Management and Informatics,

Hokkaido Information University, 59-2 Nishi-Nopporo,

Ebetsu, Hokkaido 069-8585, Japan

e-mail: h-satoh@do-johodai.ac.jp

H. Satoh · H. Tsutui

Department of Cardiovascular Medicine, Hokkaido University

Graduate School of Medicine, Sapporo, Japan

S. Fujii

Department of Molecular and Cellular Pathobiology

and Therapeutics, Nagoya City University Graduate School

of Pharmaceutical Sciences, Nagoya, Japan ratios (95\% confidence interval) of the groups with $<6$ and $\geq 8 \mathrm{~h}$ for hypertension was $2.43(1.40-4.20, P<0.01)$ and $2.28(1.31-3.95, P<0.01)$, respectively, adjusted for conventional cardiovascular risk factors.

Conclusion The present study demonstrates that both long and short habitual sleep duration were significantly associated with high blood pressure values and hypertension occurrence in Japanese male subjects.

Keywords Sleep duration - Blood pressure . Hypertension - Japanese male subjects - Epidemiology

\section{Introduction}

Sleep loss, long-term sleep deprivation, and alterations in sleep duration are common in modern society [1], with evidence showing that we are sleeping on average only $6.8 \mathrm{~h}$ per night, which is $1.5 \mathrm{~h}$ less than we did 100 years ago [2]. Change of sleep condition exerts deleterious effects on detectable changes in metabolic [3, 4], endocrine [5], and sympathetic tone [6]. These findings suggest that alterations of habitual sleep duration may predispose to overt change of blood pressure values. Several previous studies demonstrated that shorter sleep duration was related to hypertension, compared with subjects with $7 \mathrm{~h}$ of sleep per night in Caucasian populations [7-10]. The first US National Health and Nutrition Examination Survey (NHANES-I) elucidated that sleeping $5 \mathrm{~h}$ or less per night was associated with a $60 \%$ increased risk of incident hypertension in Caucasian middle-aged subjects during a mean follow-up of 8-10 years [10]. The Sleep Heart Health Study demonstrated that US subjects sleeping more than $9 \mathrm{~h}$ per night had $30 \%$ higher prevalence of hypertension as compared with those sleeping between 7 and $8 \mathrm{~h}$ 
per night [7]. The relationship between sleep duration and hypertension occurrence in previous studies has been controversial; however, this association among Japanese general population has not been fully investigated. Moreover, the relationship between sleep duration and blood pressure values remains unanswered.

The purpose of the present study is to examine crosssectional associations of sleep duration with blood pressure values and hypertension occurrence in Japanese male subjects.

\section{Materials and methods}

\section{Study subjects}

The study subjects consisted of 1,886 food and beverage company male employees, aged from 38 from 58 years, who had their annual health examinations during the period from April 2010 to March 2011. A total of 216 subjects were excluded for the following reasons: medication of hypertension $(n=201)$, medication of sleep disturbance $(n=12)$, and medication of depression $(n=3)$. Thus, 1,670 male subjects were enrolled in the present study. The study protocol was approved by the ethical committee of Hokkaido Information University, and written informed consent was obtained from each subject.

\section{Baseline data measurement}

All study subjects were asked to complete a self-questionnaire that included smoking habit, alcohol consumption, frequency of exercise, family history of hypertension, and medical history. The questionnaire was distributed to the subjects in advance of their annual health check-up and was collected at the examination. The subjects who had never smoked and ex-smokers were classified as "non-smokers." Drinkers were defined as those who consumed alcohol once per week or more. "Exercise" subjects were defined as those who regularly exercised more than once per week. Hypertension, dyslipidemia, and diabetes mellitus were defined as receiving medical agents at the health examination. Body mass index (BMI) was calculated as body weight (kilograms) divided by squared height (meters). Blood pressure was measured by a trained nurse using a standard mercury sphygmomanometer, with the study subjects in sitting position after at least a 5-min rest. A blood sample was obtained from the antecubital vein in the morning after an overnight fast, and serum was separated. After precipitation by heparin-manganese, total cholesterol and highdensity lipoprotein (HDL)-cholesterol were measured by the phosphotungstate method. Triglyceride was measured enzymatically. Glucose was enzymatically determined by the hexokinase method.

\section{Definition of hypertension}

Incident hypertension subjects were defined as those with systolic blood pressure $\geq 140 \mathrm{mmHg}$ and/or diastolic blood pressure $\geq 90 \mathrm{mmHg}$ at their health examination.

Statistical analysis

The study subjects were categorized into four groups according to sleep duration: $<6,6-, 7-$, and $\geq 8 \mathrm{~h}$. The characteristics of the study subjects were expressed as mean \pm standard deviation (SD) for continuous variables, median (interquartile range) for skewed distribution variables, and percentages for categorical variables according to sleep duration. The differences of variables among groups were examined by analysis of variance (ANOVA), Kruskal-Wallis test, or chi-square test. Next, Bonferroni procedure was used between two groups as a post hoc test. The association between sleep duration and hypertension was assessed by using multiple logistic regression analysis. The principle model included candidate variables of age, BMI, smoking, alcohol, exercise, dyslipidemia, diabetes mellitus, family history of hypertension, values such as systolic blood pressure, total cholesterol, triglyceride, HDL-cholesterol, and glucose. $P$ value less than 0.05 was considered to indicate statistical significance. All statistical analyses were performed using the SPSS statistical package for Windows version 11.0 (Chicago, IL, USA).

\section{Results}

Table 1 presents the characteristics of study subjects according to sleep duration levels. The mean age and BMI of the study subjects was $50 \pm 6$ years and $23.8 \pm 2.8 \mathrm{~kg} / \mathrm{m}^{2}$, respectively. The numbers $(\%)$ of the study subjects with sleep duration of $<6,6-, 7$-, and $\geq 8$ h were 201 (12.0), 628 (37.6), 638 (38.2), and 203 (12.2), respectively. Variables such as age, BMI, alcohol, exercise, family history of hypertension, triglyceride, and HDL-cholesterol were significantly different among the groups. Subjects with 7-h sleep duration were older and more likely to be nondrinkers.

Figure 1 shows systolic (a) and diastolic (b) blood pressure values according to sleep duration levels. The mean systolic and diastolic blood pressure values of the study subjects were $119 \pm 15$ and $74 \pm 11 \mathrm{mmHg}$, respectively. The mean systolic blood pressure values with sleep duration $<6$, 6-, 7-, and $\geq 8 \mathrm{~h}$ were $122 \pm 14,119 \pm 15$, $118 \pm 15$, and $122 \pm 17 \mathrm{mmHg}$, respectively ( $P$ for trend $<0.01)$. The mean diastolic blood pressure values with sleep 
Table 1 Characteristics of the study subjects according to sleep duration levels

\begin{tabular}{|c|c|c|c|c|c|c|}
\hline & \multirow[t]{2}{*}{ Total $(n=1,670)$} & \multicolumn{4}{|l|}{ Sleep duration } & \multirow[t]{2}{*}{$P$ value } \\
\hline & & $<6(n=201)$ & 6- $(n=628)$ & $7-(n=638)$ & $\geq 8(n=203)$ & \\
\hline Age (years) & $50 \pm 6$ & $48 \pm 5$ & $49 \pm 6$ & $51 \pm 6$ & $51 \pm 5$ & $<0.001$ \\
\hline Body mass index $\left(\mathrm{kg} / \mathrm{m}^{2}\right)$ & $23.8 \pm 2.8$ & $24.4 \pm 3.1$ & $23.8 \pm 2.8$ & $23.6 \pm 2.6$ & $23.8 \pm 2.8$ & $<0.05$ \\
\hline Smoking (\%) & 52.2 & 54.7 & 52.1 & 51.7 & 51.5 & 0.90 \\
\hline Alcohol (\%) & 74.3 & 74.1 & 73.6 & 71.2 & 85.2 & $<0.01$ \\
\hline Exercise $(\%)$ & 28.2 & 20.9 & 31.7 & 27.2 & 27.9 & $<0.05$ \\
\hline Dyslipidemia (\%) & 4.0 & 4.0 & 3.5 & 3.8 & 6.4 & 0.32 \\
\hline Diabetes mellitus (\%) & 3.2 & 1.5 & 3.8 & 3.3 & 2.5 & 0.38 \\
\hline Family history of hypertension (\%) & 15.6 & 21.4 & 16.9 & 14.6 & 9.4 & $<0.01$ \\
\hline Total cholesterol (mg/dL) & $208 \pm 33$ & $206 \pm 36$ & $208 \pm 31$ & $208 \pm 34$ & $208 \pm 33$ & 0.90 \\
\hline Triglyceride $(\mathrm{mg} / \mathrm{dL})$ & $106(76-150)$ & $104(76-150)$ & $127(87-173)$ & $105(73-146)$ & $104(75-152)$ & $<0.05$ \\
\hline HDL-cholesterol (mg/dL) & $57 \pm 14$ & $56 \pm 13$ & $57 \pm 15$ & $56 \pm 14$ & $59 \pm 15$ & $<0.05$ \\
\hline Glucose (mg/dL) & $96 \pm 20$ & $94 \pm 12$ & $96 \pm 19$ & $97 \pm 20$ & $97 \pm 24$ & 0.13 \\
\hline
\end{tabular}

Values are mean $\pm \mathrm{SD}$, median (interquartile range), and percentage

$H D L$ high-density lipoprotein

(a) Systolic blood pressure

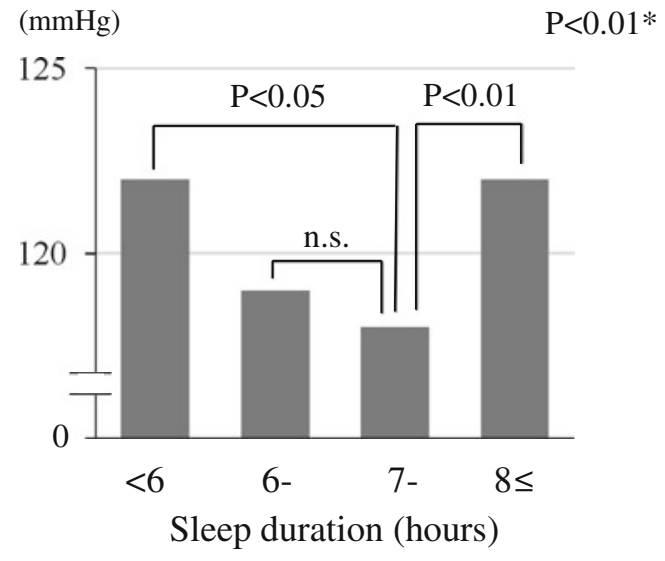

(b) Diastolic blood pressure

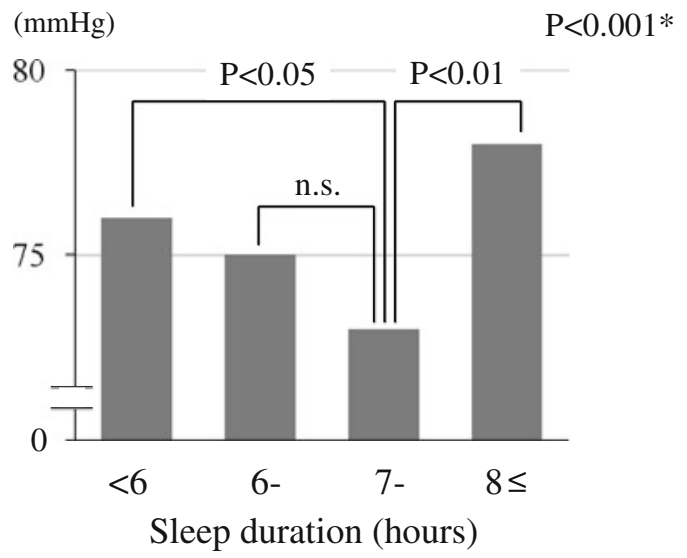

Fig. 1 Systolic (a) and diastolic (b) blood pressure values according to sleep duration levels. $* P$ for trend duration $<6$, 6-, 7-, and $\geq 8 \mathrm{~h}$ were $76 \pm 11,75 \pm 10$, $73 \pm 11$, and $78 \pm 12 \mathrm{mmHg}$, respectively $(P$ for trend $<0.001)$. Compared with subjects with sleep duration of $7 \mathrm{~h}$ as a referent, those with sleep duration of both $<6$ and $\geq 8 \mathrm{~h}$ had significantly greater systolic and diastolic blood pressure values.

Table 2 presents multivariate predictor variables for hypertension by multiple logistic regression analysis. The number $(\%)$ of study subjects with hypertension was 139 (8.3\%). Risk factors such as age, BMI, smoking, alcohol, family history of hypertension, and glucose were significant and independent predictors for hypertension.

Table 3 presents the odds ratio and $95 \%$ confidence interval (CI) for hypertension according to sleep duration levels. The numbers $(\%)$ of hypertensive subjects with sleep duration $<6,6-, 7$-, and $\geq 8$ h were 27 (13.4), 36 (5.7), 48 (7.5), and 28 (13.8), respectively. On multivariate analysis with sleep duration of $7 \mathrm{~h}$ as the reference, the adjusted odds ratio for subjects with sleep duration $<6 \mathrm{~h}$ for hypertension was 2.43 (95\% CI 1.40-4.20, $P<0.01$ ), and for sleep duration $\geq 8$ h was 2.28 (95\% CI $1.31-3.95, P<0.01)$.

\section{Discussion}

The present study demonstrates that sleep duration of both $<6$ and $\geq 8 \mathrm{~h}$ are significantly associated with high blood pressure values and hypertension occurrence in Japanese male subjects.

Bonnet et al. [1] reported that nearly one-third of adults had sleep duration of less than $6 \mathrm{~h}$ per night, and sleep 
Table 2 Multivariate predictor variables for hypertension by multiple logistic regression analysis

\begin{tabular}{lccc}
\hline & $\begin{array}{l}\text { Odds } \\
\text { ratio }\end{array}$ & $95 \%$ CI & $P$ value \\
\hline Age (per 1 year increase) & 1.04 & $1.01-1.08$ & $<0.05$ \\
$\begin{array}{l}\text { Body mass index (per } 1 \mathrm{~kg} / \mathrm{m}^{2} \\
\text { increase) }\end{array}$ & 1.17 & $1.09-1.25$ & $<0.001$ \\
Smoking & 1.87 & $1.26-2.78$ & $<0.01$ \\
Alcohol & 2.23 & $1.31-3.77$ & $<0.01$ \\
Family history of hypertension & 1.78 & $1.13-2.79$ & $<0.05$ \\
Glucose (per $10 \mathrm{mg} / \mathrm{dL}$ increase) & 1.15 & $1.07-1.23$ & $<0.001$ \\
\hline
\end{tabular}

CI confidence interval

Table 3 Odds ratio and $95 \%$ CI for hypertension according to sleep duration levels

\begin{tabular}{cllll}
\hline \multicolumn{5}{c}{ Sleep duration $(\mathrm{h})$} \\
\cline { 2 - 5 } & $\begin{array}{l}<6 \\
(n=201)\end{array}$ & $\begin{array}{l}6- \\
(n=628)\end{array}$ & $\begin{array}{l}7- \\
(n=638)\end{array}$ & $\begin{array}{l}\geq 8 \\
(n=203)\end{array}$ \\
\hline $\begin{array}{c}\text { Hypertension, } \\
n(\%)\end{array}$ & $27(13.4)$ & $36(5.7)$ & $48(7.5)$ & $28(13.8)$ \\
Unadjusted & & & & \\
Odds ratio & 2.73 & 1.33 & 1.00 & 2.45 \\
$95 \%$ CI & $1.60-4.64$ & $0.84-2.10$ & - & $1.45-4.15$ \\
$P$ value & $<0.001$ & 0.23 & - & $<0.01$ \\
Model $1^{\text {a }}$ & & & & \\
Odds ratio & 2.49 & 1.28 & 1.00 & 2.40 \\
$95 \%$ CI & $1.43-4.33$ & $0.80-2.05$ & - & $1.40-4.12$ \\
$P$ value & $<0.01$ & 0.30 & - & $<0.01$ \\
Model 2 & & & & \\
Odds ratio & 2.43 & 1.27 & 1.00 & 2.28 \\
$95 \%$ CI & $1.40-4.20$ & $0.81-1.99$ & - & $1.31-3.95$ \\
$P$ value & $<0.01$ & 0.30 & - & $<0.01$ \\
\hline
\end{tabular}

$C I$ confidence interval, $H D L$ high-density lipoprotein

a Adjusted for age, body mass index, smoking, alcohol, exercise, and family history of hypertension

b Model 1 + adjusted for diabetes mellitus, dyslipidemia, total cholesterol, triglyceride, HDL-cholesterol, and glucose

deficiency, long-term sleep deprivation, and alterations in sleep duration have become common in modern society. The quality and duration of sleep have been indicated as factors affecting health condition in general population [11, 12]. In epidemiologic studies, compared with subjects with habitual sleep duration of $7 \mathrm{~h}$, short and long sleep durations were related to increased BMI [13, 14], development of diabetes mellitus $[15,16]$, and occurrence of cardiovascular disease [17-19]. Recent epidemiological cohort studies indicated that short and long sleep duration were also closely associated with mortality [20, 21]. Ikehara et al. [22] demonstrated that both shorter and longer sleep duration were associated with increased mortality due to all causes for both genders, yielding a U-shaped relationship with total mortality with a nadir at 7-h sleep duration from a large-scale prospective study of 98,634 Japanese men and women in the Japan Collaborative Cohort Study. However, the relationship between sleep duration and blood pressure values in Japanese general population has not been fully established.

Previous studies indicated that sleep disorders might play a crucial role in determining blood pressure values both in the office and over the $24 \mathrm{~h}$ and modulating the day-night blood pressure profile [23, 24], which could affect the prognosis of hypertensive patients $[25,26]$. Recent studies demonstrated that both increased and reduced sleep duration may be related to increased risk of cardiovascular disease and hypertension [22, 27]. These studies clarified that sleep duration defined as acutely induced sleep deprivation was closely associated with blood pressure values; however, the relationship between habitual sleep duration and blood pressure values has still been controversial [28].

Previous studies reported that short habitual sleep duration increased blood pressure values in general population [29]; however, this relationship might differ among ages and ethnicity $[7,30]$. The present study demonstrated that short habitual sleep duration was significantly associated with high blood pressure values and hypertension occurrence in Japanese middle-aged male subjects, which confirmed the previous results. The biological mechanisms mediating the association of short habitual sleep duration with development and occurrence of hypertension are uncertain; however, short habitual sleep duration may disrupt circadian rhythm and autonomic balance [30,31]. The alterations in these functions might shift the daily blood pressure profile to higher values, lead to a nondipping pattern, increase blood pressure variability, and induce disturbances in the diurnal rhythm of cardiac output [32,33].

The present study also demonstrated that long habitual sleep duration was significantly associated with high blood pressure values and hypertension occurrence. The rate of drinkers was more likely to be higher in subjects with sleep duration $\geq 8$ than $<6,6-$, and $7 \mathrm{~h}$ (Table 1), and the alcohol covariate was a significant and independent predictor for hypertension (Fig. 1). Sesso et al. [34] indicated that light to moderate alcohol consumption exhibited a linear association with increasing risk for hypertension development. Nakanishi et al. [35] demonstrated that the risk for hypertension increased according to the increment of alcohol consumption doses in Japanese middle-aged male subjects. Thus, these results may be a potential causal basis for the result in the present study. Patel et al. [36] reported that $\mathrm{C}$-reactive protein and interleukin-6 levels rose with increasing habitual sleep duration. These inflammatory mediators might also increase the risk of hypertension in subjects with long habitual sleep [37]. 
The limitations of the present study are as follows. First, sleep duration obtained by self-questionnaire might differ from precisely measured sleep duration. However, Lockely et al. [38] reported that good agreement was found between self-reported sleep duration and values obtained through actigraphic monitoring. Second, the present study examined blood pressure values once, in the health examination. The accuracy of blood pressure values would likely have little effect on the results of this analysis. Third, the present study was a cross-sectional observation study. The possibility of unmeasured confounding variables, such as sedentary lifestyle and obstructive sleep apnea, could not be excluded.

In conclusion, we demonstrated that both short and long habitual sleep duration were significantly associated with high blood pressure values and hypertension occurrence in Japanese male subjects. Finally, it is important to note that adequate sleep duration should be one of the important strategies for hypertension occurrence.

Conflict of interest The authors declare that they have no conflict of interest.

\section{References}

1. Bonnet MH, Arand DL. We are chronically sleep deprived. Sleep. 1995;18:908-11.

2. National Sleep Foundation. Sleep in America Poll 2003. Washington, DC: National Sleep Foundation; 2003.

3. Knutson KL, Spiegel K, Penev P, Van Cauter E. The metabolic consequences of sleep deprivation. Sleep Med Rev. 2007;11: 163-78.

4. Spiegel K, Tasali E, Leproult R, Van Cauter E. Effects of poor and short sleep on glucose metabolism and obesity risk. Nat Rev Endocrinol. 2009;5:253-61.

5. Spiegel K, Leproult R, L'Hermite-Baleriaux M, Copinschi G, Penev PD, Van Cauter E. Leptin levels are dependent on sleep duration: relationships with sympathovagal balance, carbohydrate regulation, cortisol, and thyrotropin. J Clin Endocrinol Metab. 2004;89:5762-71.

6. Spiegel K, Leproult R, Van Cauter E. Impact of sleep debt on metabolic and endocrine function. Lancet. 1999;354:1435-9.

7. Gottlieb DJ, Redline S, Nieto FJ, Baldwin CM, Newman AB, Resnick HE, et al. Association of usual sleep duration with hypertension: the Sleep Heart Health Study. Sleep. 2006;29:1009-14.

8. Cappuccio FP, Stranges S, Kandala NB, Miller MA, Taggart FM, Kumari $M$, et al. Gender-specific associations of short sleep duration with prevalent and incident hypertension: the Whitehall II Study. Hypertension. 2007;50:693-700.

9. Stang A, Moebus S, Mohlenkamp S, Erbel R, Jockel KH. Genderspecific associations of short sleep duration with prevalent hypertension. Hypertension. 2008;51:e15-6. author reply e17.

10. Staessen JA, Thijs L, Fagard R, O'Brien ET, Clement D, de Leeuw PW, et al. Predicting cardiovascular risk using conventional vs ambulatory blood pressure in older patients with systolic hypertension. Systolic Hypertension in Europe Trial Investigators. JAMA. 1999;282:539-46.

11. Kapur VK, Redline S, Nieto FJ, Young TB, Newman AB, Henderson JA. The relationship between chronically disrupted sleep and healthcare use. Sleep. 2002;25:289-96.
12. Shankar A, Charumathi S, Kalidindi S. Sleep duration and selfrated health: the national health interview survey 2008. Sleep. 2011;34:1173-7.

13. Stranges S, Cappuccio FP, Kandala NB, Miller MA, Taggart FM, Kumari M, et al. Cross-sectional versus prospective associations of sleep duration with changes in relative weight and body fat distribution: the Whitehall II Study. Am J Epidemiol. 2008;167: 321-9.

14. Patel SR, Hu FB. Short sleep duration and weight gain: a systematic review. Obesity (Silver Spring). 2008;16:643-53.

15. Ayas NT, White DP, Al-Delaimy WK, Manson JE, Stampfer MJ, Speizer FE, et al. A prospective study of self-reported sleep duration and incident diabetes in women. Diabetes Care. 2003;26:380-4.

16. Yaggi HK, Araujo AB, McKinlay JB. Sleep duration as a risk factor for the development of type 2 diabetes. Diabetes Care. 2006;29:657-61.

17. Greenland P, Knoll MD, Stamler J, Neaton JD, Dyer AR, Garside $\mathrm{DB}$, et al. Major risk factors as antecedents of fatal and nonfatal coronary heart disease events. JAMA. 2003;290:891-7.

18. Sabanayagam C, Shankar A. Sleep duration and cardiovascular disease: results from the National Health Interview Survey. Sleep. 2010;33:1037-42.

19. Shankar A, Koh WP, Yuan JM, Lee HP, Yu MC. Sleep duration and coronary heart disease mortality among Chinese adults in Singapore: a population-based cohort study. Am J Epidemiol. 2008;168:1367-73.

20. Rod NH, Vahtera J, Westerlund H, Kivimaki M, Zins M, Goldberg $\mathrm{M}$, et al. Sleep disturbances and cause-specific mortality: results from the GAZEL cohort study. Am J Epidemiol. 2011;173:300-9.

21. Kripke DF, Langer RD, Elliott JA, Klauber MR, Rex KM. Mortality related to actigraphic long and short sleep. Sleep Med. 2011;12:28-33.

22. Ikehara S, Iso H, Date C, Kikuchi S, Watanabe Y, Wada Y, et al. Association of sleep duration with mortality from cardiovascular disease and other causes for Japanese men and women: the JACC study. Sleep. 2009;32:295-301.

23. Loredo JS, Nelesen R, Ancoli-Israel S, Dimsdale JE. Sleep quality and blood pressure dipping in normal adults. Sleep. 2004;27:1097-103.

24. Friedman O, Logan AG. Nocturnal blood pressure profiles among normotensive, controlled hypertensive and refractory hypertensive subjects. Can J Cardiol. 2009;25:e312-6.

25. Li Y, Boggia J, Thijs L, Hansen TW, Kikuya M, BjorklundBodegard $\mathrm{K}$, et al. Is blood pressure during the night more predictive of cardiovascular outcome than during the day? Blood Press Monit. 2008;13:145-7.

26. Fagard RH, Thijs L, Staessen JA, Clement DL, De Buyzere ML, De Bacquer DA. Night-day blood pressure ratio and dipping pattern as predictors of death and cardiovascular events in hypertension. J Hum Hypertens. 2009;23:645-53.

27. Eguchi K, Pickering TG, Schwartz JE, Hoshide S, Ishikawa J, Ishikawa $\mathrm{S}$, et al. Short sleep duration as an independent predictor of cardiovascular events in Japanese patients with hypertension. Arch Intern Med. 2008;168:2225-31.

28. Stranges S, Dorn JM, Shipley MJ, Kandala NB, Trevisan M, Miller MA, et al. Correlates of short and long sleep duration: a cross-cultural comparison between the United Kingdom and the United States: the Whitehall II Study and the Western New York Health Study. Am J Epidemiol. 2008;168:1353-64.

29. Kim J, Jo I. Age-dependent association between sleep duration and hypertension in the adult Korean population. Am J Hypertens. 2010;23:1286-91.

30. Gangwisch JE, Heymsfield SB, Boden-Albala B, Buijs RM, Kreier F, Pickering TG, et al. Short sleep duration as a risk factor 
for hypertension: analyses of the first National Health and Nutrition Examination Survey. Hypertension. 2006;47:833-9.

31. Folkow B. "Structural factor" in primary and secondary hypertension. Hypertension. 1990;16:89-101.

32. Folkow B. Mental stress and its importance for cardiovascular disorders; physiological aspects, "from-mice-to-man". Scand Cardiovasc J. 2001;35:163-72.

33. Coca A. Circadian rhythm and blood pressure control: physiological and pathophysiological factors. J Hypertens Suppl. 1994;12:S13-21.

34. Sesso HD, Cook NR, Buring JE, Manson JE, Gaziano JM. Alcohol consumption and the risk of hypertension in women and men. Hypertension. 2008;51:1080-7.
35. Nakanishi N, Yoshida H, Nakamura K, Suzuki K, Tatara K. Alcohol consumption and risk for hypertension in middle-aged Japanese men. J Hypertens. 2001;19:851-5.

36. Patel SR, Zhu X, Storfer-Isser A, Mehra R, Jenny NS, Tracy R, et al. Sleep duration and biomarkers of inflammation. Sleep. 2009;32:200-4.

37. Patel SR. Sleep—an affair of the heart. Sleep. 2009;32:289-90.

38. Lockley SW, Skene DJ, Arendt J. Comparison between subjective and actigraphic measurement of sleep and sleep rhythms. J Sleep Res. 1999;8:175-83. 